Communications in Physics, Vol. 20, No. 3 (2010), pp. 277-288

\title{
PHASE TRANSITION IN BINARY MIXTURE OF BOSE GASES
}

\author{
TRAN HUU PHAT \\ Vietnam Atomic Energy Commission \\ and \\ Dong Do University \\ LE VIET HOA \\ Hanoi National University of Education \\ NGUYEN TUAN ANH \\ Institute for Nuclear Science and Technique \\ NGUYEN VAN LONG \\ Institute for Nuclear Science and Technique \\ and \\ Gialai Teacher College
}

\begin{abstract}
Based on the Cornwall-Jackiw-Tomboulis (CJT) effective action approach a theoretical formalism is established for studying the Bose-Einstein condensation (BEC) in a binary mixture of Bose gases. The effective potential, which preserves the Goldstone theorem, is found in the Hartree-Fock (HF) approximation. This quantity is then used to consider the equation of state (EOS) and the phase transition of the system.
\end{abstract}

\section{INTRODUCTION}

In recent years there appears a lot of experimental works dealing with BEC of systems composed of two distinct species of atoms [1-10]. The typical experiments were performed with atoms of ${ }^{87} \mathrm{Rb}$ in two different hyperfine states $\left|F=1, m_{f}=-1\right\rangle$ and $\left|F=2, m_{f}=1\right\rangle$, which behave as two completely distinguishable species [1] because the hyperfine splitting is much larger than any other relevant energy scale in the system. The multicomponent BEC is not a simple extension of the single component BEC. There arise many novel phenomena such as the quantum tunnelling of spin domain [5], vortex configuration [2], phase segregation of BEC mixture [10] and so on. Moreover, it is worth mentioning that the most special feature of all experiments realizing BEC in dilute Bose gases is that almost every parameter of the system is controlable. In connection with experimental efforts there are theoretical progresses [11-20] aiming at describing different observed phenomena of multicomponent systems as well as testing various models and methods, which have been commonly employed to consider phase transitions of relativistic theories. 
In the present article, a theoretical formalism for studying BEC in the global $U(1) \times U(1)$ model is formulated by means of the CJT effective action [21] combining with the gapless HF resummation [22]. We then obtain the effective potential in the HF approximation, which respects the Goldstone theorem.

The paper is organized as follows. In Section II we derive the desired effective potential. Section III is devoted to the physical property study of binary mixture. The conclusion and outlook are presented in section IV.

\section{EFFECTIVE POTENTIAL IN HF APPROXIMATION}

Let us begin with the idealized binary mixture of Bose gases given by the Lagrangian

$$
\begin{aligned}
£= & \phi^{*}\left(-i \frac{\partial}{\partial t}-\frac{\nabla^{2}}{2 m_{\phi}}\right) \phi+\psi^{*}\left(-i \frac{\partial}{\partial t}-\frac{\nabla^{2}}{2 m_{\psi}}\right) \psi \\
& -\mu_{1} \phi^{*} \phi+\frac{\lambda_{1}}{2}\left(\phi^{*} \phi\right)^{2}-\mu_{2} \psi^{*} \psi+\frac{\lambda_{2}}{2}\left(\psi^{*} \psi\right)^{2}+\frac{\lambda}{2}\left(\phi^{*} \phi\right)\left(\psi^{*} \psi\right),
\end{aligned}
$$

where $\mu_{1}\left(\mu_{2}\right)$ represents the chemical potential of the field $\phi(\psi), m_{1}\left(m_{2}\right)$ the mass of $\phi$ atom ( $\psi$ atom), and $\lambda_{1}, \lambda_{2}$ and $\lambda$ the coupling constants. The boundedness of the potential requires that $4 \lambda_{1} \lambda_{2}-\lambda^{2}>0$ for repulsive self-interactions, $\lambda_{1}>0, \lambda_{2}>0$. This constraint ensures the stability of mixture of condensates in experimental realization.

In the tree approximation the condensate densities $\phi_{0}^{2}$ and $\psi_{0}^{2}$ correspond to local minimum of the potential. They fulfill

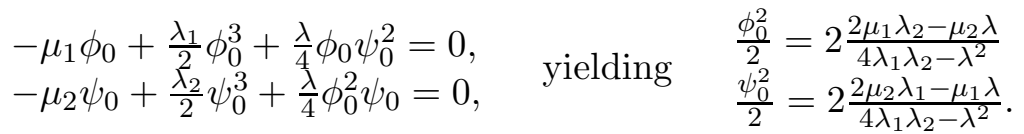

Now let us focus on the calculation of effective potential in HF approximation. At first the field operators $\phi$ and $\psi$ are decomposed

$$
\phi=\frac{1}{\sqrt{2}}\left(\phi_{0}+\phi_{1}+i \phi_{2}\right), \quad \psi=\frac{1}{\sqrt{2}}\left(\psi_{0}+\psi_{1}+i \psi_{2}\right)
$$

Inserting (3) into (1) we get, among others, the interaction Lagrangian

$$
\begin{aligned}
£_{i n t} & =\left(\frac{\lambda_{1}}{2} \phi_{0} \phi_{1}+\frac{\lambda}{4} \psi_{0} \psi_{1}\right)\left(\phi_{1}^{2}+\phi_{2}^{2}\right)+\frac{\lambda_{1}}{8}\left(\phi_{1}^{2}+\phi_{2}^{2}\right)^{2} \\
& +\left(\frac{\lambda_{2}}{2} \psi_{0} \psi_{1}+\frac{\lambda}{4} \phi_{0} \phi_{1}\right)\left(\psi_{1}^{2}+\psi_{2}^{2}\right)+\frac{\lambda_{2}}{8}\left(\psi_{1}^{2}+\psi_{2}^{2}\right)^{2} \\
& +\frac{\lambda}{8}\left(\phi_{1}^{2}+\phi_{2}^{2}\right)\left(\psi_{1}^{2}+\psi_{2}^{2}\right),
\end{aligned}
$$


and the inverse propagators in the tree approximation

$$
\begin{aligned}
& D_{0}^{-1}(k)=\left(\begin{array}{cc}
\frac{\vec{k}^{2}}{2 m_{\phi}}-\mu_{1}+\frac{3 \lambda_{1}}{2} \phi_{0}^{2}+\frac{\lambda}{4} \psi_{0}^{2} & -\omega \\
\omega & \frac{\vec{k}^{2}}{2 m_{\phi}}-\mu_{1}+\frac{\lambda_{1}}{2} \phi_{0}^{2}+\frac{\lambda}{4} \psi_{0}^{2}
\end{array}\right) \\
& G_{0}^{-1}(k)=\left(\begin{array}{cc}
\frac{\vec{k}^{2}}{2 m_{\psi}}-\mu_{2}+\frac{3 \lambda_{2}}{2} \psi_{0}^{2}+\frac{\lambda}{4} \phi_{0}^{2} & -\omega \\
\omega & \frac{\vec{k}^{2}}{2 m_{\psi}}-\mu_{2}+\frac{\lambda_{2}}{2} \psi_{0}^{2}+\frac{\lambda}{4} \phi_{0}^{2}
\end{array}\right) .
\end{aligned}
$$

From (2) and (4) it follows that

$$
\begin{aligned}
& E_{\phi}=+\sqrt{\left(\frac{\vec{k}^{2}}{2 m_{\phi}}+\lambda_{1} \phi_{0}^{2}\right) \frac{\vec{k}^{2}}{2 m_{\phi}}} \rightarrow E_{\phi} \approx \pm k \sqrt{\frac{\lambda_{1} \phi_{0}^{2}}{2 m_{\phi}}} \quad \text { for small } k \\
& E_{\psi}=+\sqrt{\left(\frac{\vec{k}^{2}}{2 m_{\psi}}+\lambda_{2} \psi_{0}^{2}\right) \frac{\vec{k}^{2}}{2 m_{\psi}}} \rightarrow E_{\psi} \approx \pm k \sqrt{\frac{\lambda_{2} \psi_{0}^{2}}{2 m_{\psi}}} \quad \text { for small } k
\end{aligned}
$$

which are the Bogoliubov dispersion relations for binary mixture of Bose gases in the broken phase associating with Goldstone bosons due to $U(1) \times U(1)$ breaking.

Assuming the ansatz [23]

$$
D^{-1}=\left(\begin{array}{cc}
\frac{\vec{k}^{2}}{2 m_{\phi}}+M_{1}^{2} & -\omega \\
\omega & \frac{\vec{k}^{2}}{2 m_{\phi}}+M_{3}^{2}
\end{array}\right), \quad G^{-1}=\left(\begin{array}{cc}
\frac{\vec{k}^{2}}{2 m_{\psi}}+M_{2}^{2} & -\omega \\
\omega & \frac{\vec{k}^{2}}{2 m_{\psi}}+M_{4}^{2}
\end{array}\right)
$$

for inverse propagators $\mathrm{D}, \mathrm{G}$ which clearly show that the Goldstone theorem fails in the HF approximation. In order to restore it, let us invoke the method developped in [22], which in our case is achieved by adding a correction $\Delta V$ to the CJT effective potential $V_{\beta}^{C J T}$, namely,

$$
\begin{aligned}
\tilde{V}_{\beta}^{C J T} & =V_{\beta}^{C J T}+\Delta V \\
\Delta V_{\beta}^{C J T} & =\frac{a \lambda_{1}}{2}\left[2 P_{a b} P_{b a}-P_{a a} P_{b b}\right]+\frac{b \lambda_{2}}{2}\left[2 Q_{a b} Q_{b a}-Q_{a a} Q_{b b}\right]+\frac{c \lambda}{2} P_{a a} Q_{b b} \\
P_{a b} & =\int_{\beta} D_{a b} ; \quad Q_{a b}=\int_{\beta} G_{a b} .
\end{aligned}
$$

It is easily checked that choosing $a=b=-1 / 2$ and $c=0$ we are led to effective potential $\tilde{V}_{\beta}^{C J T}$ obeying the requirements imposed in [22]. Indeed, substituting these values of a, b 
and $\mathrm{c}$ into (6) and (7) it is found that

$$
\begin{aligned}
\tilde{V}_{\beta}^{C J T}\left(\phi_{0}, \psi_{0}, D, G\right)= & -\frac{\mu_{1}}{2} \phi_{0}^{2}+\frac{\lambda_{1}}{8} \phi_{0}^{4}-\frac{\mu_{2}}{2} \psi_{0}^{2}+\frac{\lambda_{2}}{8} \psi_{0}^{4}+\frac{\lambda}{8} \phi_{0}^{2} \psi_{0}^{2}+\frac{1}{2} \int_{\beta} \operatorname{tr}\left\{\ln D^{-1}(k)\right. \\
& \left.+\ln G^{-1}(k)+\left[D_{0}^{-1}\left(k ; \phi_{0}, \psi_{0}\right) D\right]+\left[G_{0}^{-1}\left(k ; \phi_{0}, \psi_{0}\right) G\right]-2 \mathbb{1}\right\} \\
& +\frac{\lambda_{1}}{8} P_{11}^{2}+\frac{\lambda_{1}}{8} P_{22}^{2}+\frac{3 \lambda_{1}}{4} P_{11} P_{22}+\frac{\lambda_{2}}{8} Q_{11}^{2}+\frac{\lambda_{2}}{8} Q_{22}^{2}+\frac{3 \lambda_{2}}{4} Q_{11} Q_{22} \\
& +\frac{\lambda}{8} P_{11} Q_{11}+\frac{\lambda}{8} P_{11} Q_{22}+\frac{\lambda}{8} P_{22} Q_{11}+\frac{\lambda}{8} P_{22} Q_{22} .
\end{aligned}
$$

Since $\tilde{V}_{\beta}^{C J T}$ contains divergent integrals corresponding to zero temperature contributions we must proceed to the regularization. To this end, we make use of the dimensional regularization by performing momentum integration in $d=3-\epsilon$ dimensions and then taking $\epsilon \rightarrow 0$, the regularized integrals turn out to be finite [25]. By this way, we obtain the effective potential consisting only finite terms. From (8), we deduce immediately the following equations:

a- The gap equations

$$
-\mu_{1}+\frac{\lambda_{1}}{2} \phi_{0}^{2}+\frac{\lambda}{4} \psi_{0}^{2}+\Sigma_{2}^{\phi}=0, \quad-\mu_{2}+\frac{\lambda}{4} \phi_{0}^{2}+\frac{\lambda_{2}}{2} \psi_{0}^{2}+\Sigma_{2}^{\psi}=0 .
$$

At critical temperatures of BEC (see Section IV) we have $\phi_{0}=\psi_{0}=0$, and Eqs (9) give $\mu_{1}=\Sigma_{2}^{\phi}, \mu_{2}=\Sigma_{2}^{\psi}$, which manifest exactly the Hugenholz-Pines theorem [26] extended to binary mixture.

b- The SD equations

$$
\begin{array}{ll}
D^{-1}=D_{0}^{-1}(k)+\Sigma^{\phi}, & \Sigma^{\phi}=\left(\begin{array}{cc}
\Sigma_{1}^{\phi} & 0 \\
0 & \Sigma_{2}^{\phi}
\end{array}\right), \\
G^{-1}=G_{0}^{-1}(k)+\Sigma^{\psi}, & \Sigma^{\psi}=\left(\begin{array}{cc}
\Sigma_{1}^{\psi} & 0 \\
0 & \Sigma_{2}^{\psi}
\end{array}\right),
\end{array}
$$

in which

$$
\begin{array}{ll}
\Sigma_{1}^{\phi}=\frac{\lambda_{1}}{2} P_{11}+\frac{3 \lambda_{1}}{2} P_{22}+\frac{\lambda}{4} Q_{11}+\frac{\lambda}{4} Q_{22}, & \Sigma_{2}^{\phi}=\frac{3 \lambda_{1}}{2} P_{11}+\frac{\lambda_{1}}{2} P_{22}+\frac{\lambda}{4} Q_{11}+\frac{\lambda}{4} Q_{22}, \\
\Sigma_{1}^{\psi}=\frac{\lambda_{2}}{2} Q_{11}+\frac{3 \lambda_{2}}{2} Q_{22}+\frac{\lambda}{4} P_{11}+\frac{\lambda}{4} P_{22}, & \Sigma_{2}^{\psi}=\frac{3 \lambda_{2}}{2} Q_{11}+\frac{\lambda_{2}}{2} Q_{22}+\frac{\lambda}{4} P_{11}+\frac{\lambda}{4} P_{22}
\end{array}
$$

and

$$
M_{1}^{2}=-\mu_{1}+\frac{3 \lambda_{1}}{2} \phi_{0}^{2}+\frac{\lambda}{4} \psi_{0}^{2}+\Sigma_{1}^{\phi}, \quad M_{2}^{2}=-\mu_{2}+\frac{3 \lambda_{2}}{2} \psi_{0}^{2}+\frac{\lambda}{4} \phi_{0}^{2}+\Sigma_{1}^{\psi}
$$


Combining (9) and (10) we get the forms for inverse propagators

$$
D^{-1}=\left(\begin{array}{cc}
\frac{\vec{k}^{2}}{2 m_{\phi}}+M_{1}^{2} & -\omega \\
\omega & \frac{\vec{k}^{2}}{2 m_{\phi}}
\end{array}\right) ; \quad G^{-1}=\left(\begin{array}{cc}
\frac{\vec{k}^{2}}{2 m_{\psi}}+M_{2}^{2} & -\omega \\
\omega & \frac{\vec{k}^{2}}{2 m_{\psi}}
\end{array}\right) .
$$

It is obvious that the dispersion relations related to (11) read

$$
\begin{aligned}
& E_{\phi}=\sqrt{\frac{\vec{k}^{2}}{2 m_{\phi}}\left(\frac{\vec{k}^{2}}{2 m_{\phi}}+M_{1}^{2}\right)} \longrightarrow \sqrt{\frac{M_{1}^{2}}{2 m_{\phi}}} k \text { as } k \rightarrow 0, \\
& E_{\psi}=\sqrt{\frac{\vec{k}^{2}}{2 m_{\psi}}\left(\frac{\vec{k}^{2}}{2 m_{\phi}}+M_{2}^{2}\right)} \longrightarrow \sqrt{\frac{M_{2}^{2}}{2 m_{\psi}}} k \text { as } k \rightarrow 0,
\end{aligned}
$$

which express the Goldstone theorem. Due to the Landau criteria for superfluidity [27] the binary mixture turns out to be superfluid in broken phase and speeds of sound in each condensate are given respectively by

$$
C_{\phi}=\sqrt{\frac{M_{1}^{2}}{2 m_{\phi}}}, \quad C_{\psi}=\sqrt{\frac{M_{2}^{2}}{2 m_{\psi}}} .
$$

Ultimately the one-particle-irreducible effective potential $\tilde{V}_{\beta}^{C J T}\left(\phi_{0}, \psi_{0}\right)$ is

$$
\begin{aligned}
\tilde{V}_{\beta}^{C J T}\left(\phi_{0}, \psi_{0}\right)= & -\frac{\mu_{1}}{2} \phi_{0}^{2}+\frac{\lambda_{1}}{8} \phi_{0}^{4}-\frac{\mu_{2}}{2} \psi_{0}^{2}+\frac{\lambda_{2}}{8} \psi_{0}^{4}+\frac{\lambda}{8} \phi_{0}^{2} \psi_{0}^{2}+\frac{1}{2} \int_{\beta} \operatorname{tr}\left\{\ln D^{-1}(k)+\ln G^{-1}(k)\right\} \\
& -\frac{\lambda_{1}}{8} P_{11}^{2}-\frac{\lambda_{1}}{8} P_{22}^{2}-\frac{3 \lambda_{1}}{4} P_{11} P_{22}-\frac{\lambda_{2}}{8} Q_{11}^{2}-\frac{\lambda_{2}}{8} Q_{22}^{2}-\frac{3 \lambda_{2}}{4} Q_{11} Q_{22} \\
& -\frac{\lambda}{8} P_{11} Q_{11}-\frac{\lambda}{8} P_{11} Q_{22}-\frac{\lambda}{8} P_{22} Q_{11}-\frac{\lambda}{8} P_{22} Q_{22} .
\end{aligned}
$$

\section{PHYSICAL PROPERTIES}

\section{III.1. Equations of State}

Let us now consider EOS starting from the effective potential. To this end, we begin with the pressure defined by

$$
P=-\left.\tilde{V}_{\beta}^{C J T}\left(\phi_{0}, \psi_{0}, D, G\right)\right|_{\text {at minimum }},
$$

from which the total particle densities are determined

$$
\rho_{i}=\frac{\partial P}{\partial \mu_{i}}, \quad i=1,2 .
$$

Taking into account the fact that derivatives of $\tilde{V}_{\beta}^{C J T}\left(\phi_{0}, \psi_{0}, D, G\right)$ with respect to its arguments vanish at minimum we get

$$
\rho_{1}=\frac{\partial V_{\beta}^{C J T}}{\partial \mu_{1}}=\frac{\phi_{0}^{2}}{2}+\frac{P_{11}}{2}+\frac{P_{22}}{2}, \quad \rho_{2}=\frac{\partial V_{\beta}^{C J T}}{\partial \mu_{2}}=\frac{\psi_{0}^{2}}{2}+\frac{Q_{11}}{2}+\frac{Q_{22}}{2} .
$$


Hence, the gap equations (9) become

$$
\mu_{1}=\lambda_{1} \rho_{1}+\frac{\lambda}{2} \rho_{2}+\lambda_{1} P_{11}, \quad \mu_{2}=\lambda_{2} \rho_{2}+\frac{\lambda}{2} \rho_{1}+\lambda_{2} Q_{11},
$$

and the particle densities in condensates are

$$
\frac{\phi_{0}^{2}}{2}=\rho_{1}-\frac{P_{11}}{2}-\frac{P_{22}}{2}, \quad \frac{\psi_{0}^{2}}{2}=\rho_{2}-\frac{Q_{11}}{2}-\frac{Q_{22}}{2} .
$$

The differences

$$
\begin{aligned}
& \rho_{1}^{e}=\rho_{1}-\rho_{1}^{c}=\frac{P_{11}}{2}+\frac{P_{22}}{2}, \\
& \rho_{2}^{e}=\rho_{2}-\rho_{2}^{c}=\frac{Q_{11}}{2}+\frac{Q_{22}}{2},
\end{aligned}
$$

represents the densities of particles in excited states with $k \neq 0$.

Combining Eqs. (11), (13) and (14) together produces the following expression for the pressure

$$
\begin{aligned}
P= & \frac{\lambda_{1}}{2} \rho_{1}^{2}+\frac{\lambda_{2}}{2} \rho_{2}^{2}+\frac{\lambda}{2} \rho_{1} \rho_{2}-\frac{1}{2} \int_{\beta} \operatorname{tr}\left\{\ln D^{-1}(k)+\ln G^{-1}(k)\right\} \\
& -\frac{\lambda_{1}}{2} P_{11}^{2}-\frac{\lambda_{2}}{2} Q_{11}^{2}+\lambda_{1} \rho_{1} P_{11}+\lambda_{2} \rho_{2} Q_{11} .
\end{aligned}
$$

The free energy follows from the Legendre transform

$$
E=\mu_{1} \rho_{1}+\mu_{2} \rho_{2}-P
$$

and reads

$$
E=\frac{\lambda_{1}}{2} \rho_{1}^{2}+\frac{\lambda_{2}}{2} \rho_{2}^{2}+\frac{\lambda}{2} \rho_{1} \rho_{2}+\frac{1}{2} \int_{\beta} \operatorname{tr}\left\{\ln D^{-1}(k)+\ln G^{-1}(k)\right\}+\frac{\lambda_{1}}{2} P_{11}^{2}+\frac{\lambda_{2}}{2} Q_{11}^{2} .
$$

Eqs. (17) and (18) constitute the EOS governing all thermodynamical processes, in particular, phase transitions of the binary mixture, which is a two-component system with two conserved charges.

To proceed further it is interesting to consider the high temperature regime, $T / \mu_{i} \gg$ 1 , associating with symmetry restoration/nonrestoration (SR/SNR) and inverse symmetry breaking (ISB), which are the main subject of the next section. In Appendix B are listed the high temperature expansions of all integrals appearing in $V_{\beta}$ and related quantities. From (17) we find the pressure to first order in $\lambda_{1}, \lambda_{2}$ and $\lambda$ for temperature just below the critical temperature

$$
P=\frac{\lambda_{1} \rho_{1}^{2}+\lambda_{2} \rho_{2}^{2}+\lambda \rho_{1} \rho_{2}}{2}+\frac{\left(m_{\phi}^{3 / 2}+m_{\psi}^{3 / 2}\right) \zeta(5 / 2)}{2 \sqrt{2} \pi^{3 / 2}} T^{5 / 2}+\frac{\left(m_{\phi}^{3} \lambda_{1}+m_{\psi}^{3} \lambda_{2}\right)[\zeta(3 / 2)]^{2}}{16 \pi^{3}} T^{3},
$$

which reduces to the well-known result of Lee and Yang for single component Bose gas [28] without invoking the double counting subtraction as was done in Ref. [29]. Based on the formula

$$
E=-\frac{\partial}{\partial \beta}[\beta P(\mu)]_{\mu}, \quad \beta=1 / T
$$


the high temperature behaviour of the free energy density is also derived in the same approximation

$$
\begin{aligned}
E=- & \frac{1}{2}\left(\lambda_{1} \rho_{1}^{2}+\lambda_{2} \rho_{2}^{2}+\lambda \rho_{1} \rho_{2}\right)-\frac{3\left(m_{\phi}^{3 / 2} \lambda_{1} \rho_{1}+m_{\psi}^{3 / 2} \lambda_{2} \rho_{2}\right) \zeta(3 / 2)}{4 \sqrt{2} \pi^{3 / 2}} T^{3 / 2} \\
& +\frac{3\left(m_{\phi}^{3 / 2}+m_{\psi}^{3 / 2}\right) \zeta(5 / 2)}{4 \sqrt{2} \pi^{3 / 2}} T^{5 / 2}+\frac{\left(m_{\phi}^{3} \lambda_{1}+m_{\psi}^{3} \lambda_{2}\right)[\zeta(3 / 2)]^{2}}{8 \pi^{3}} T^{3}
\end{aligned}
$$

Let us remark that the preceding expression for $E$ does not reduce to the corresponding one given in Ref. [29] for single component Bose gas because the approximation taken there is different from ours.

Next the low temperature regime, $T / \mu_{i} \ll 1$, is concerned. We are able to write the low temperature behaviours of the equations for $M_{1}^{2}$ and $M_{2}^{2}$ as follows

$$
\begin{aligned}
& M_{1}^{2}=2 \lambda_{1} \rho_{1}-\frac{2 \sqrt{2} M_{1}^{3} m_{\phi}^{3 / 2} \lambda_{1}}{3 \pi^{2}}-\frac{2 \sqrt{2} m_{\phi}^{3} \lambda_{1} \pi^{2}}{15 M_{1}^{5}} T^{4}, \\
& M_{2}^{2}=2 \lambda_{2} \rho_{2}-\frac{2 \sqrt{2} M_{2}^{3} m_{\psi}^{3 / 2} \lambda_{2}}{3 \pi^{2}}-\frac{2 \sqrt{2} m_{\psi}^{3} \lambda_{2} \pi^{2}}{15 M_{2}^{5}} T^{4},
\end{aligned}
$$

which require a self-consistent solution for $M_{1}^{2}$ and $M_{2}^{2}$ as functions of densities and temperature. The first approximation we can choose is

$$
M_{1}^{2} \simeq 2 \lambda_{1} \rho_{1}, \quad M_{2}^{2} \simeq 2 \lambda_{2} \rho_{2}
$$

Substituting respectively (19) into (17) we arrive at the low temperature dependences of chemical potentials and pressure

$$
\begin{aligned}
& \mu_{1}=\lambda_{1} \rho_{1}+\frac{\lambda}{2} \rho_{2}+\frac{4 m_{\phi}^{3 / 2} \lambda_{1}^{5 / 2} \rho_{1}^{3 / 2}}{3 \pi^{2}}+\frac{m_{\phi}^{3 / 2} \pi^{2}}{60 \lambda_{1}^{3 / 2} \rho_{1}^{5 / 2}} T^{4} \\
& \mu_{2}=\lambda_{2} \rho_{2}+\frac{\lambda}{2} \rho_{1}+\frac{4 m_{\psi}^{3 / 2} \lambda_{2}^{5 / 2} \rho_{2}^{3 / 2}}{3 \pi^{2}}+\frac{m_{\psi}^{3 / 2} \pi^{2}}{60 \lambda_{2}^{3 / 2} \rho_{2}^{5 / 2}} T^{4},
\end{aligned}
$$

and

$$
\begin{aligned}
P= & \frac{\lambda_{1} \rho_{1}^{2}+\lambda_{2} \rho_{2}^{2}+\lambda \rho_{1} \rho_{2}}{2}+\frac{4\left(m_{\phi}^{3 / 2} \lambda_{1}^{5 / 2} \rho_{1}^{5 / 2}+m_{\psi}^{3 / 2} \lambda_{2}^{5 / 2} \rho_{2}^{5 / 2}\right)}{5 \pi^{2}} \\
& +\left(\frac{m_{\phi}^{3 / 2}}{\lambda_{1}^{3 / 2} \rho_{1}^{3 / 2}}+\frac{m_{\psi}^{3 / 2}}{\lambda_{2}^{3 / 2} \rho_{2}^{3 / 2}}\right) \frac{\pi^{2} T^{4}}{36}-\left(\frac{m_{\phi}^{3}}{\rho_{1}}+\frac{m_{\psi}^{3}}{\rho_{2}}\right) \frac{T^{4}}{45}-\frac{8\left(m_{\phi}^{3} \lambda_{1}^{4} \rho_{1}^{3}+m_{\psi}^{3} \lambda_{2}^{4} \rho_{2}^{3}\right)}{9 \pi^{2}} \\
& -\left(\frac{m_{\phi}^{3}}{\lambda_{1}^{4} \rho_{1}^{5}}+\frac{m_{\psi}^{3}}{\lambda_{2}^{4} \rho_{2}^{5}}\right) \frac{\pi^{4} T^{8}}{7200} .
\end{aligned}
$$

It is worth to mention that Eq. (20) does not coincide with the low temperature expansion of pressure for one-component Bose gas [29] because several T-dependent terms were 
missed in that work. Accordingly we get the equation for free energy

$$
\begin{aligned}
E=\mu_{1} \rho_{1}+\mu_{2} \rho_{2}-P= & \frac{\lambda_{1} \rho_{1}^{2}+\lambda_{2} \rho_{2}^{2}+\lambda \rho_{1} \rho_{2}}{2}+\frac{8\left(m_{\phi}^{3 / 2} \lambda_{1}^{5 / 2} \rho_{1}^{5 / 2}+m_{\psi}^{3 / 2} \lambda_{2}^{5 / 2} \rho_{2}^{5 / 2}\right)}{15 \pi^{2}} \\
& -\left(\frac{m_{\phi}^{3 / 2}}{\lambda_{1}^{3 / 2} \rho_{1}^{3 / 2}}+\frac{m_{\psi}^{3 / 2}}{\lambda_{2}^{3 / 2} \rho_{2}^{3 / 2}}\right) \frac{\pi^{2} T^{4}}{90}+\left(\frac{m_{\phi}^{3}}{\rho_{1}}+\frac{m_{\psi}^{3}}{\rho_{2}}\right) \frac{T^{4}}{45} \\
& +\frac{8\left(m_{\phi}^{3} \lambda_{1}^{4} \rho_{1}^{3}+m_{\psi}^{3} \lambda_{2}^{4} \rho_{2}^{3}\right)}{9 \pi^{4}}+\left(\frac{m_{\phi}^{3}}{\lambda_{1}^{4} \rho_{1}^{5}}+\frac{m_{\psi}^{3}}{\lambda_{2}^{4} \rho_{2}^{5}}\right) \frac{\pi^{2} T^{8}}{7200} .
\end{aligned}
$$

\section{III.2. Symmetry Non Restoration and Inverse Symmetry Breaking}

Introducing the effective chemical potentials

$$
\bar{\mu}_{1}=\mu_{1}-\Sigma_{2}^{\phi}, \quad \bar{\mu}_{2}=\mu_{2}-\Sigma_{2}^{\psi},
$$

the gap equations (9) can be rewritten as

$$
\frac{\lambda_{1}}{2} \phi_{0}^{2}+\frac{\lambda}{4} \psi_{0}^{2}=\bar{\mu}_{1}, \quad \frac{\lambda}{4} \phi_{0}^{2}+\frac{\lambda_{2}}{2} \psi_{0}^{2}=\bar{\mu}_{2},
$$

which yield

$$
\frac{\phi_{0}^{2}}{2}=2 \frac{2 \bar{\mu}_{1} \lambda_{2}-\bar{\mu}_{2} \lambda}{4 \lambda_{1} \lambda_{2}-\lambda^{2}} ; \quad \frac{\psi_{0}^{2}}{2}=2 \frac{2 \bar{\mu}_{2} \lambda_{1}-\bar{\mu}_{1} \lambda}{4 \lambda_{1} \lambda_{2}-\lambda^{2}} .
$$

Eqs. (21) resemble (2) with $\mu_{i}$ replaced by $\bar{\mu}_{i}$.

It is evident that the symmetry breaking in $\phi$ sector is restored at $T=T_{c 1}$ if

$$
\phi_{0}^{2}=0 \quad \text { or } \quad 2 \lambda_{2} \bar{\mu}_{1}\left(T_{c 1}\right)-\lambda \bar{\mu}_{2}\left(T_{c 1}\right)=0 .
$$

A similar process occurs in $\psi$ sector at $T=T_{c 2}$ if

$$
\psi_{0}^{2}=0 \quad \text { or } \quad 2 \lambda_{1} \bar{\mu}_{2}\left(T_{c 2}\right)-\lambda \bar{\mu}_{1}\left(T_{c 2}\right)=0 .
$$

Taking into account the high temperature expansions for $\bar{\mu}_{1}$ and $\bar{\mu}_{2}$ Eqs. (22) and (23) provide the approximate formulae for the critical temperatures $T_{c 1}$ and $T_{c 2}$

$$
\begin{aligned}
& T_{c 1}=2 \pi\left[\frac{2\left(\lambda \mu_{2}-2 \lambda_{2} \mu_{1}\right)}{\left(m_{\phi}^{3 / 2} \lambda^{2}+m_{\psi}^{3 / 2} \lambda \lambda_{2}-8 m_{\phi}^{3 / 2} \lambda_{1} \lambda_{2}\right) \zeta(3 / 2)}\right]^{2 / 3}, \\
& T_{c 2}=2 \pi\left[\frac{2\left(\lambda \mu_{1}-2 \lambda_{1} \mu_{2}\right)}{\left(m_{\psi}^{3 / 2} \lambda^{2}+m_{\phi}^{3 / 2} \lambda \lambda_{1}-8 m_{\psi}^{3 / 2} \lambda_{1} \lambda_{2}\right) \zeta(3 / 2)}\right]^{2 / 3},
\end{aligned}
$$

which suggest several scenarios for symmetry restoration (SR), symmetry non restoration(SNR) and inverse symmetry breaking (ISB) in our model, which were discussed in 
detail in Ref. [30]. Regarding the influence of thermal effect on the manifestation of SNB/ISB let us estimate the temperature dependent coupling constants determined by

$$
\begin{aligned}
\lambda_{1}(T) & =\left.\frac{1}{3} \frac{\delta^{4} \tilde{V}_{\beta}^{C J T}\left(\phi_{0}, \psi_{0}\right)}{\delta \phi_{0}^{4}}\right|_{\phi_{0}=\psi_{0}=0}, \\
\lambda_{2}(T) & =\left.\frac{1}{3} \frac{\delta^{4} \tilde{V}_{\beta}^{C J T}\left(\phi_{0}, \psi_{0}\right)}{\delta \psi_{0}^{4}}\right|_{\phi_{0}=\psi_{0}=0}, \\
\lambda(T) & =\left.\frac{1}{2} \frac{\delta^{4} \tilde{V}_{\beta}^{C J T}\left(\phi_{0}, \psi_{0}\right)}{\delta \phi_{0}^{2} \delta \psi_{0}^{2}}\right|_{\phi_{0}=\psi_{0}=0} .
\end{aligned}
$$

Using the high temperature expansion for $\tilde{V}_{\beta}$ from Eqs. (25) we are led to the following expressions for the effective coupling constants $\lambda_{1}(T), \lambda_{2}(T)$ and $\lambda(T)$ up to the cubic order of $\lambda_{1}, \lambda_{2}$ and $\lambda$

$$
\begin{aligned}
& \lambda_{1}(T)=\left.\frac{1}{3} \frac{\delta^{4} \tilde{V}^{C J T}}{\delta \phi_{0}^{4}}\right|_{\phi_{0}=0}=\lambda_{1}-\frac{3 \zeta(1 / 2) \sqrt{T}}{8 \sqrt{2} \pi^{3 / 2}}\left(m_{\psi}^{3 / 2} \lambda^{2}+9 m_{\phi}^{3 / 2} \lambda_{1}^{2}\right) \\
& +\frac{[\zeta(1 / 2)]^{2} T}{16 \pi^{3}}\left(\frac{3 m_{\phi}^{3 / 2} m_{\psi}^{3 / 2}}{2} \lambda^{2} \lambda_{1}+m_{\psi}^{3} \lambda^{2} \lambda_{2}+9 m_{\phi}^{3} \lambda_{1}^{3}\right) \\
& -\frac{9 \zeta(3 / 2) T^{3 / 2}}{64 \pi^{7 / 2} \sqrt{-\mu_{1}+\frac{T^{3 / 2}\left(m_{\psi}^{3 / 2} \lambda+4 m_{\phi}^{3 / 2} \lambda_{1}\right) \zeta(3 / 2)}{(2 \pi)^{3 / 2}}}}\left(m_{\phi}^{3 / 2} m_{\psi}^{3 / 2} \lambda \lambda_{1}^{2}+4 m_{\phi}^{3} \lambda_{1}^{3}\right) \\
& -\frac{\zeta(3 / 2) T^{3 / 2}}{64 \pi^{7 / 2} \sqrt{-\mu_{2}+\frac{T^{3 / 2}\left(m_{\phi}^{3 / 2} \lambda+4 m_{\psi}^{3 / 2} \lambda_{2}\right) \zeta(3 / 2)}{(2 \pi)^{3 / 2}}}}\left(m_{\phi}^{3 / 2} m_{\psi}^{3 / 2} \lambda^{3}+4 m_{\psi}^{3} \lambda^{2} \lambda_{2}\right) \text {, } \\
& \lambda_{2}(T)=\left.\frac{1}{3} \frac{\delta^{4} \tilde{V}^{C J T}}{\delta \psi_{0}^{4}}\right|_{\psi_{0}=0}=\lambda_{2}-\frac{3 \zeta(1 / 2) \sqrt{T}}{8 \sqrt{2} \pi^{3 / 2}}\left(m_{\phi}^{3 / 2} \lambda^{2}+9 m_{\psi}^{3 / 2} \lambda_{2}^{2}\right) \\
& +\frac{[\zeta(1 / 2)]^{2} T}{16 \pi^{3}}\left(\frac{3 m_{\phi}^{3 / 2} m_{\psi}^{3 / 2}}{2} \lambda^{2} \lambda_{2}+m_{\phi}^{3} \lambda^{2} \lambda_{1}+9 m_{\psi}^{3} \lambda_{2}^{3}\right) \\
& -\frac{9 \zeta(3 / 2) T^{3 / 2}}{64 \pi^{7 / 2} \sqrt{-\mu_{2}+\frac{T^{3 / 2}\left(m_{\phi}^{3 / 2} \lambda+4 m_{\psi}^{3 / 2} \lambda_{2}\right) \zeta(3 / 2)}{(2 \pi)^{3 / 2}}}}\left(m_{\phi}^{3 / 2} m_{\psi}^{3 / 2} \lambda \lambda_{2}^{2}+4 m_{\psi}^{3} \lambda_{2}^{3}\right) \\
& -\frac{\zeta(3 / 2) T^{3 / 2}}{64 \pi^{7 / 2} \sqrt{-\mu_{1}+\frac{T^{3 / 2}\left(m_{\psi}^{3 / 2} \lambda+4 m_{\phi}^{3 / 2} \lambda_{1}\right) \zeta(3 / 2)}{(2 \pi)^{3 / 2}}}}\left(m_{\phi}^{3 / 2} m_{\psi}^{3 / 2} \lambda^{3}+4 m_{\phi}^{3} \lambda^{2} \lambda_{1}\right) \text {, }
\end{aligned}
$$




$$
\begin{aligned}
\lambda(T)= & \left.2 \frac{\delta^{4} \tilde{V}^{C J T}}{\delta \phi_{0}^{2} \psi_{0}^{2}}\right|_{\phi_{0}=0, \psi_{0}=0}=\lambda-\frac{9 \zeta(1 / 2) \sqrt{T}}{4 \sqrt{2} \pi^{3 / 2}}\left(m_{\phi}^{3 / 2} \lambda \lambda_{1}+m_{\psi}^{3 / 2} \lambda \lambda_{2}\right) \\
& +\frac{[\zeta(1 / 2)]^{2} T}{32 \pi^{3}}\left(m_{\phi}^{3 / 2} m_{\psi}^{3 / 2} \lambda^{3}+9 m_{\phi}^{3 / 2} m_{\psi}^{3 / 2} \lambda \lambda_{1} \lambda_{2}+12 m_{\phi}^{3} \lambda \lambda_{1}^{2}+12 m_{\psi}^{3} \lambda \lambda_{2}^{2}\right) \\
& -\frac{3 \zeta(3 / 2) T^{3 / 2}}{32 \pi^{7 / 2} \sqrt{-\mu_{1}+\frac{T^{3 / 2}\left(m_{\psi}^{3 / 2} \lambda+4 m_{\phi}^{3 / 2} \lambda_{1}\right) \zeta(3 / 2)}{(2 \pi)^{3 / 2}}}}\left(m_{\phi}^{3 / 2} m_{\psi}^{3 / 2} \lambda^{2} \lambda_{1}+4 m_{\phi}^{3} \lambda \lambda_{1}^{2}\right) \\
& -\frac{3 \zeta(3 / 2) T^{3 / 2}}{32 \pi^{7 / 2} \sqrt{-\mu_{2}+\frac{T^{3 / 2}\left(m_{\phi}^{3 / 2} \lambda+4 m_{\psi}^{3 / 2} \lambda_{2}\right) \zeta(3 / 2)}{(2 \pi)^{3 / 2}}}}\left(m_{\phi}^{3 / 2} m_{\psi}^{3 / 2} \lambda^{2} \lambda_{2}+4 m_{\psi}^{3} \lambda \lambda_{2}^{2}\right) .
\end{aligned}
$$

Eqs. (26) prove that the effective coupling constants strongly depend on temperature. This is quite different from the relativistic case $[22,28]$.

It is known that in comparison with single component system phase transition in twocomponent one is much more involved. The fact is that, in addition to the phase transition caused by the mechanical instability taking place in one-component system, there exist in binary mixture the diffusive instabilities [31,32]. As was pointed out in Ref. [32] in order to determine the state of two-component body it is necessary to specify three quantities, for instance, $P, T$ and the concentration fraction $y$ which is defined as

$$
y=\rho_{1} / \rho, \quad \rho=\rho_{1}+\rho_{2} .
$$

For symmetrical reason, we need to consider only $0<y<0.5$. Then the condition for mechanical stability states that

$$
\rho\left(\frac{\partial P}{\partial \rho}\right)_{T, y} \geq 0
$$

and the constraints for diffusive stabilities read

$$
\left(\frac{\partial \mu_{1}}{\partial y}\right)_{T, P} \geq 0 \quad \text { or } \quad\left(\frac{\partial \mu_{2}}{\partial y}\right)_{T, P} \leq 0 .
$$

The numerical study for phase transition in binary mixture of Bose gases will be reported in a forthcoming publication.

\section{CONCLUSION AND OUTLOOK}

Due to growing interest in binary mixture of Bose gases we studied a non-relativistic model of two-component complex field. Our main goal is to formulate a theoretical formalism for this physical system. To this end, with the aid of the CJT approach we established the finite CJT effective potential, which preserves the Goldstone theorem in broken phase. This is our major success. The expression for the pressure, which depends on particle densities, was derived by means of the fact that the pressure is determined by the effective potential at minimum. As a consequence, the free energy was obtained straightforwardly. 
The EOS at low and high temperatures were considered. In particular, the critical temperatures were determined, which generated various scenarios for SR, SNR/ISB with some constraints on coupling constants. It was proved that, unfortunately, these constraints were altered strongly by thermal effect. In order to understand better the specific properties of phase transition patterns in two-component Bose gases further study would be carried out by means of numerical computation.

It is expected that the formalism developed in this work could be fruitfully applied to a realistic investigation of trapped two-component Bose gases, in which the nonhomogeneity is incorporated into consideration.

\section{ACKNOWLEDGMENTS}

This paper was financially supported by the Vietnam National Fund for Scientific Research.

\section{REFERENCES}

[1] C. J. Myatt, E. A. Burt, R. W. Ghrist, E. A. Cornell, and C. E. Wieman, Phys. Rev. Lett. 78 (1997) 586.

[2] M. R. Matthews, B. P. Anderson, P. C. Haljan, D. S. Hall, C. E. Wieman, and E. A. Cornell, Phys. Rev. Lett. 83 (1999) 2498.

[3] D. S. Hall, M. R. Matthews, J. R. Ensher, C. E. Wieman, and E. A. Cornell, Phys. Rev. Lett. 81 (1998) 1539.

[4] D. M. Stamper-Kurn, H. -J. Miesner, A. P. Chikkatur, S. Inouye, J. Stenger, and W. Ketterle, Phys. Rev. Lett. 83 (1999) 661.

[5] H. -J. Miesner, D. M. Stamper-Kurn, J. Stenger, S. Inouye, A. P. Chikkatur, and W. Ketterle, Phys. Rev. Lett. 82 (1999) 2228.

[6] G. Modugno, M. G. Modugno, F. Riboli, G. Roati, and M. Inguscio, Phys. Rev. Lett. 89 (2002) 190404.

[7] S. B. Papp, and C. E. Wieman Phys. Rev. Lett. 97 (2006) 180404.

[8] S. B. Papp, J. M. Pino, and C. E. Wieman, arXiv: 0802.2591.

[9] G. Thalhammer, G. Barontini, L. De Sarlo, J. Catani, F. Minardi, and M. Inguscio, arXiv: 0803.2763.

[10] J. Stenger, S. Inouye, D. M. Stemper-Kurn, H. -J. Miesner, A. P. Chikkatur, and W. Ketterle, Nature (London). 396 (1998) 345.

[11] T-L. Ho, and V. B. Shenoy, Phys. Rev. Lett. 77 (1996) 3276.

[12] C. K. Law, H. Pu, N. P. Bigelow, and J. H. Eberly, Phys. Rev. Lett. 79 (1997) 3105.

[13] B. D. Esry, C. H. Greene, Phys. Rev. A57 (1998) 1265.

[14] R. Graham, and D. Walls, Phys. Rev. A57 (1998) 484.

[15] P. Ao, and S. T. Chui, J. Phys. B: At. Mol. Opt. Phys. 33 (2000) 535.

[16] I. E. Mazets, Phys. Rev. A65 (2002) 033618.

[17] D. M. Jezek, and P. Capuzzi, Phys. Rev. A66 (2002) 015602.

[18] A. A. Svidzinsky, and S. T. Chui, Phys. Rev. A68 (2003) 013612.

[19] S. G. Bhongale, and E. Timmermans, arXiv: 0711.4007.

[20] B. van Schaeybroeck, arXiv: 0805.2512.

[21] J. M. Cornwall, R. Jackiw, and E. Tomboulis, Phys. Rev. D10 (1974) 2428; G. Amelino-Camelia and S.Y.Pi, Phys. Rev. D47 (1993) 2356.

[22] Yu. B. Ivanov, F. Riek, and J. Knoll, Phys. Rev. D71 (2005) 105016.

[23] We accept the ansatz for the full propagators assumed by M. G. Alford, M. Braby, and A. Schmitt, J. Phys. G35 (2008) 025002.

[24] Tran Huu Phat, Nguyen Tuan Anh, and Le Viet Hoa, Eur. Phys. J. A19 (2004) 359; Tran Huu Phat, Le Viet Hoa, Nguyen Tuan Anh, and Nguyen Van Long, Phys. Rev. D76 (2007) 125027; Tran Huu 
Phat, Nguyen Van Long, Nguyen Tuan Anh, and Le Viet Hoa, Kaon Condensation in Linear Sigma Model, submitted to Phys. Rev. D.

[25] J. O. Andersen, cond-mat/0305138.

[26] N. M. Hugenholz and D. Pines, Phys. Rev. 116 (1958) 489.

[27] L. Landau, and E. M. Lifshitz, Statistical Physics, Pergamon Press, 1969.

[28] T. D. Lee, and C. N. Yang, Phys. Rev. 112 (1958) 1419; Phys. Rev. 117 (1960) 897.

[29] T. Haugset, H. Haugerud, and F. Ravndal, Ann. Phys. (NY) 266 (1998) 27.

[30] M. B. Pinto, R. O. Ramos, and F. F. de Souza Cruz, Phys. Rev. A74 (2006) 033618.

[31] M. Barranco, and J. R. Buchler, Phys. Rev. C22 (1980) 1729.

[32] H. Müller, and B. D. Serot, Phys. Rev. C52 (1995) 2072 .

Received 28 September 2009. 